\section{Role of tactual-kinesthetic feedback in transfer of perceptual learning in the golden hamster*}

\author{
BARRY S. ANTON and THOMAS L. BENNETT \\ Colorado State University, Fort Collins, Colo. 80521
}

Golden hamsters were preexposed to circles and triangles in an otherwise visually sparse environment where opportunity to manipulate the forms was varied for the early experience groups. Although early experience with these stimuli enhanced their later discriminability over that shown by control animals that received no early experience, opportunity to manipulate the forms produced no additional gain in perceptual learning relative to Ss not allowed to manipulate the preexposed shapes.

Several researchers have found that early experience with stimuli enhances the later discriminability and have attempted to elucidate the mechanisms underlying this phenomenon (e.g., Bennett, Anton, \& Levitt, 1971; Bennett \& Ellis, 1968; Gibson \& Walk, 1956; Gibson, Walk, \& Tighe, 1959; Kerpelman, 1965). For example, Gibson et al (1959) found that early experience with three-dimensional stimuli facilitated their subsequent discriminability, but early experience with two-dimensional forms failed to show a comparable effect. They posit an "attention-getting" hypothesis to account for the difference. Presumably, exposure to three-dimensional stimuli enables $S$ to sort salient attributes as cues from the stimulus influx. More recently, Bennett \& Ellis (1968) have emphasized a view more in keeping with an additive theory of discrimination learning, namely, that the difference in discriminability observed between two- and three-dimensional stimuli lies in tactual-kinesthetic feedback resulting f rom m a n i p l a t i o of three-dimensional forms. Bennett and Ellis found that albino rats allowed to manipulate the preexposed forms performed better than those not allowed to manipulate in a subsequent discrimination task, thus supporting the tactual-kinesthetic hypothesis.

Bennett, Rickert, \& McAllister (1970) further examined this issue by using hooded rats with pigmented irises, an animal with a better visual system than the albino (Lashley,

*'This research was supported by a grant from the National Institute of Mental Health (MH 20076-01). Requests for reprints should be addressed to Thomas $L$. Bennett, Dept. of Psychology, Colorado State University, Fort Collins, Colo. 80521. The authors are indebted to David Benedict. Ronald Feldman, and Gregg Daniels for their aid in the conduct of this study.
1930). Their findings restricted the generality of the tactual-kinesthetic feedback hypothesis. Bennett et al found that, for rats with pigmented irises, the opportunity to manipulate the forms produced no additional gain in perceptual learning compared with Ss given no opportunity to manipulate the shapes.

The present study reexamined this issue by using golden hamsters as Ss. Since the golden hamster has pigmented irises like the hooded rat, this animal should also be less dependent upon tactual-kinesthetic feedback in learning about his environment than the albino. Consequently, tactual-kinesthetic feedback from the preexposure forms may not be required if early experience with stimuli is to enhance their later discriminability. Additionally, the present study attempted to extend the phylogenetic generality of the early experience effect.

\section{METHOD}

The Ss were golden hamsters reared from birth in a visually sparse environment (Bennett \& Ellis, 1968), with food and water available ad lib. The experiment was conducted in two stages. During Stage 1 , Ss were assigned to one of three early experience conditions: (1) in Group M $(N=11)$, the animals were allowed to manipulate the forms (three-dimensional circles and triangles covered with wire mesh) and thus receive tactual-kinesthetic feedback; (2) for Group NM $(N=8)$, the forms were still quite visible and were placed inside the cage, but manipulation of the forms was prevented by enclosing them in a wire mesh box; (3) a control Group $C(N=11)$ did not receive early e $x$ perie $n$ ce with the to-be-discriminated stimuli.

At the age of 90 days, the Ss entered Stage 2 and were given 5 days of discrimination pretraining. Pretraining consisted of training the animals to push open the panels in the absence of visual forms attached to the panels. The Ss were deprived of food for $8 \mathrm{~h}$ before each pretraining or training session. This relatively mild deprivation schedule was used because of a high incidence of death among the hamsters when more severe deprivation schedules were imposed. The testing apparatus was a Grice discrimination box as modified by Gibson \& Walk (1956).

Discrimination training was begun at the age of 95 days. The $S$ could obtain a pinch of wet mash from one of the two stimulus panels, a black circle or a black triangle on a white background covered with wire mesh, by pushing open the correct stimulus door. Half of each group was trained to circle $(t)$ and half to triangle $(+)$. The incorrect stimulus panel was locked but baited. If S's choices were correct, food was obtained and the next trial began. If the choices were incorrect, $\mathrm{S}$ was allowed to proceed into the other compartment where it was confronted with the stimuli in the same orientation. The $\mathrm{S}$ was allowed three errors per trial. Following the third error, the door in front of the correct stimulus opened and the animal was allowed to eat. The Ss were run to a criterion of 18 out of 20 correct responses, with the last 10 consecutive responses correct, or for a total of 200 trials; animals were given 10 trials per day.

RESULTS AND DISCUSSION

The principal findings of the experiment are presented in Fig. 1, which shows a plot of the percentage of correct responses as a function of successive trial blocks for the three conditions of early experience. Inspection of this graph indicates that Groups $M$ and NM did not differ appreciably in their rate of task acquisition, although early experience with stimuli did enhance their discriminability relative to that of Group C.

A Kruskal-Wallis one-way analysis of variance by ranks indicated a significant difference between the groups in terms of the mean number of trials to reach criterion $[H(2)=12.32, \mathrm{df}=2, \mathrm{p}<.02]$. To isolate the significant source of variance, Mann-Whitney U tests (two-tailed) were applied to assess differences between groups in terms of their trials to criterion. The results of these analyses are presented in Table 1. Examination of this table indicates that the performance of Groups $M$ and NM were comparable and that both of these groups learned the discrimination task in fewer trials than did Group C. Thus, early experience with stimuli significantly increased their later discriminability. 


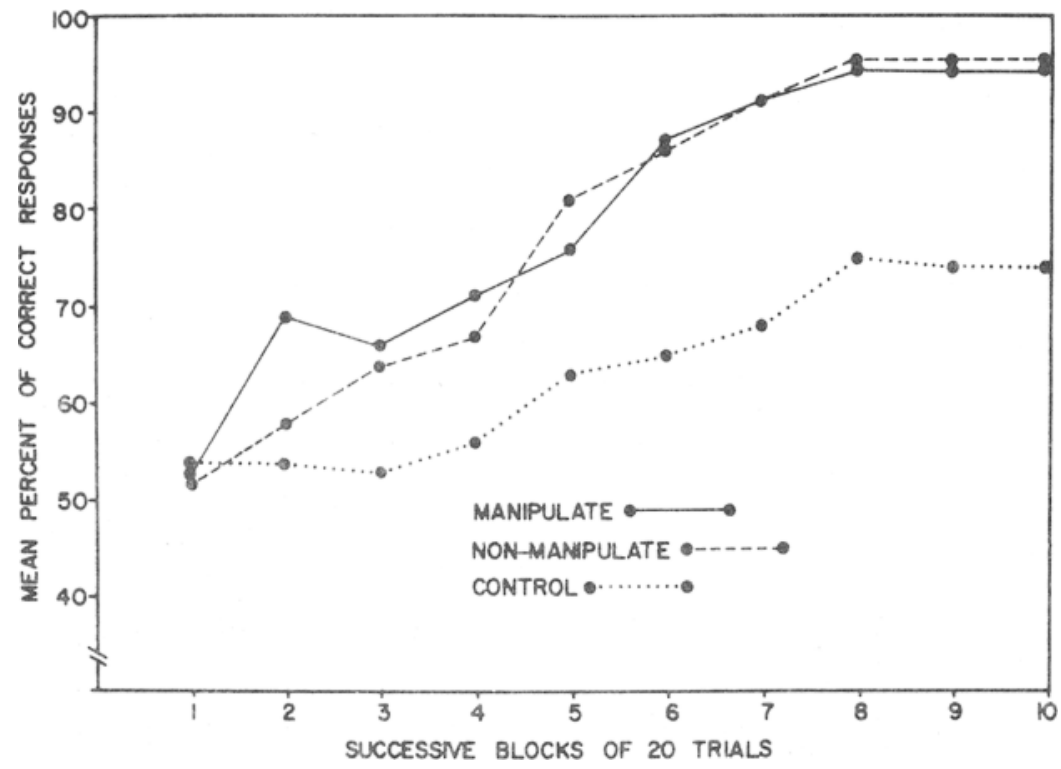

Fig. 1. Discrimination learning curves as a function of preexposure conditions.

The data also show that opportunity to manipulate the forms produced no additional gain in perceptual learning compared with that of Ss given no opportunity to manipulate the shapes.

The finding that early experience with stimuli facilitated their subsequent discriminability for the golden hamster extends phylogenetically the generality of the early experience effect. The fact that those animals receiving early stimulus experience exhibited no differences in the amount of transfer as a function of whether they received tactual-kinesthetic feedback from the forms confirms the findings of Bennett, Rickert, \& McAllister, who examined the perceptual learning

Table 1

Summary of Mann-Whitney $U$ Tests Analyzing Trials to Criterion Between the $V$ arious Stimulus Preexposure Conditions

\begin{tabular}{lrrrc}
\hline Groups & df & U & p \\
\hline M vs NM & 11.8 & & 45 & n.s. \\
M vs C & 11.11 & 12 & $p<.002$ \\
NM vs C & 8.11 & 11 & $p<.02$ \\
\hline
\end{tabular}

process in rats with pigmented irises. These studies, taken together, indicate that tactual-kinesthetic feedback from the early experience stimuli is probably only necessary for bringing about an ordering of visual cues in animals that have a relatively inferior visual system, e.g., the albino rat.

\section{REFERENCES}

BENNETT, T. L. ANTON, B. S. \& LEVITT, L. Stimulus relevancy and transfer of perceptual learning. Psychonomic Science. 1971, 25, 159-160. BENNETT, T. L.. \& ELLIS, H. C. Tactual-kinesthetic feedback from manipulation of visual forms and manipulation of visual forms and of perceptual learning. Journal of Experimental Psychology, 1968, 77, 495-500.

BENNETT, T. L., RICKERT, E. J., \& M CALLISTER, L. E. Role of tactual-kinesthetic feedback in transfer of perceptual learning for rats with pigmented irises. Perceptual \& Motor Skills, 1970, 30, 916-918.

GIBSON, E. J., \& WALK, R. D. The effect of prolonged exposure to visually presented patterns on learning to discriminate them. Journal of Comparative \& Physiological Psychology, $1956,49,239-242$.

GIBSON, E. J., WALK, R. D., \& TIGHE, T J. Enhancement and deprivation of visual stimulation during rearing as factors in visual discrimination learning. Journal of Comparative \& Physiological Psychology, 1959, 52, 72-81.

KERPELMAN, L. C. Pre-exposure to $v$ isually presented forms and non-differential reinforcement in perceptual learning. Journal of Experimental Psychology, 1965, 68, 257-262.

LASHLEY, K. S. The mechanism of vision: III. The comparative visual activity of pigmented and albino rats. Journal of Genetic Psychology, 1930, 37, 481.484. 